\title{
Client-centric Heterogeneous Access in Cognitive WLANs
}

\author{
(Invited Paper) \\ Deming Pang*, Quan Liu*, Gang Hu*, Yingwen Chen* and Ming $\mathrm{Xu}^{*}$ \\ ${ }^{*}$ Dept. of Network Engineering, Computer School \\ National University of Defense Technology \\ Changsha, China \\ Email: \{pang3724,liuquan,xuming\}@ nudt.edu.cn
}

\begin{abstract}
The spatial and temporal variations of licensed spectrum bands introduce new problems to cognitive wireless LANs. We propose a novel client-centric heterogeneous spectrum access mechanism to deal with these new challenges. All clients in a cell would not have to use the same channel to communicate with access point when there is no common available channel among these clients. In order to achieve channel agreement among nodes on different channels, a new MAC protocol is proposed with no need of common control channel. Considering that AP can only work on one channel at a time, we use a novel technique, subcarrier coding, to enable it to monitor multiple spectrum bands simultaneously. Each client can select its best channel based on local environment and requirement independently. Promising simulation results show that this new access mechanism not only resolve the problem of lacking common data channel in single cell, but also minimizes conflict significantly even in the case of a large number of clients.
\end{abstract}

\section{INTRODUCTION}

Cognitive radio techniques are proposed to enable secondary users (SUs) to opportunistically share the spectrum with licensed primary users (PUs), which have higher priority to access the licensed spectrum and must not be disturbed. The unused portions of the licensed spectrum, popularly referred to as spectrum holes, offer additional spectrum resources to SUs. However, networking on top of spectrum hole is challenging for the reason that PU can operate in any region at any time, and cause spatial and temporal variation of spectrum hole.

Spatial variation means that a channel may be available to a SU in one location, but not available to another user at a different location. The set of available channels in licensed spectrum bands mostly depends on the location of PUs, such as TV stations, cellular users, and microphones. Other than that, due to obstructions in the signal transmission path, spatial variation exists even on a smaller scale (tens of meters). Therefore, secondary users located in different areas might have different sets of available channels even they are in the same cell. To quantify this variation, a set of real-world measurements in the VHF/UHF bands have been performed in [1]. The results showed that the median number of channels available at one point but unavailable at another is close to 7 , even if these points are located in nearby buildings. Note that television stations represent the largest incumbent use of the considered UHF spectrum measured in [1], which exhibit

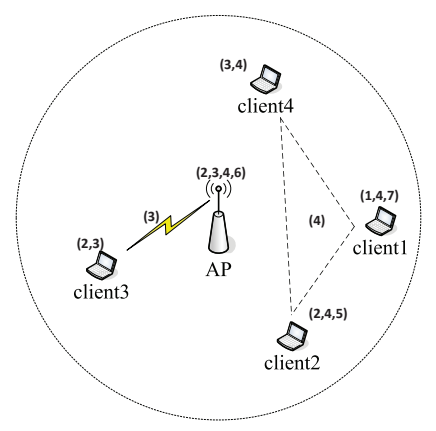

Fig. 1. Topology of considered cognitive WLAN, nodes may have different available channels due to spatial variation

moderate dynamic compared to other frequency bands, we expect more significant spatial variation in other bands (e.g., $900 \mathrm{MHz}$ GSM bands). This implies that it is not easy for an AP to select a common data channel that is available to all associated clients when we try to build a Wi-Fi like networks.

Let us consider the network presented in Fig. 1 with one $\mathrm{AP}$ and multiple associated clients. Each node in this network has its own available channel set (CS) which can be obtained by spectrum sensing or downloading from database. The CSs of each node may be different due to different locations and surroundings. For example, the CS of AP is $(2,3,4,6)$, which means that channel 2,3,4 and 6 can be used by this AP at the current time. However there is no common available channel that can be used by all clients and AP. As the coverage of an AP is expected to exceed $1 \mathrm{~km}$ or even up to tens of kilometers when an AP uses VHF/UHF bands, there could be dozens or hundreds of clients distributed around different locations. Therefore, there is high probability that an AP can not find a common idle channel among these clients.

Even if a commonly available channel in a cell exists, the temporal variation of spectrum could introduce another problem. The availability of a channel could varies along with time due to the operation of PUs. The time variation has been characterized by a number of spectrum measurements in different locations of the world [2][3]. All of the measurements indicate that the availability of spectrum changes rapidly due to the stochastic arrival and departure of PUs, as well as 
interference and noise. If all nodes in a cell use the same channel as in current $\mathrm{WiFi}$ networks, any change to that channel at just one node will make other nodes have to switch to another channel even if they can continue working on current channel. Considering every node could undergo this kind of variation, channel switching trigged by a single client could lead to the entire cell into a continuous jitter state (moving back and forth over among different channels), leaving no time for data transition.

A large number of multichannel MAC protocols have been proposed for ad hoc and infrastructure-based cognitive radio networks. To achieve channel agreement in multichannel scenario, common control channel is usually assumed in most protocols, such as HC-MAC[5], C-MAC[6] and MMAC[7]. Although they can be used to deal with the lacking common channel problem as described before in principle, the inefficiency is a big problem due to they are designed for ad hoc networks and coupled the data transmission and channel agreement. In addition, due to the lack of common control channel and the existence of bottleneck problem, we need to find a replacement to fulfill channel agreement. Other work for infrastructure-based networks, such as WhiteFi [1] and WRAN [4], has been designed to construct Wi-Fi like system. However, these systems all follows a strict master/slave relationship wherein AP performs the role of the master and select a channel for all communications in the cell. We refer to the systems, no matter WhiteFi or WRAN as homogeneous access systems since all clients in a cell use the same channel and configuration to communicate with AP.

The challenges introduced by spatial and temporal variation of spectrum holes make it inefficient to exploit spectrum with homogeneous access. Considering that there are usually multiple unused channels and secondary users are capable of adjusting radio parameters flexibly, we can not help but ask: why do we have to use the same channel in a cell? With this in mind, we propose a client-centric heterogenous media access control (CH-MAC) protocol to enable clients to use different channels simultaneously to communicate with an AP. Spectrum decision can be made by clients independently, rather than the AP as in the traditional way. Most of the prior works on multi-channel MAC in infrastructure-based networks concern on how to deal with inter-cell channel allocation, not intra-cell, and only limited studies deal with similar access. The work in [8] enables clients to access different parts of the same channel, so it still belongs to single channel access and it is essentially different from our work. The C-CSMA/CA [12] that is similar with our work enable clients in the same cell communicate with AP on different channels, but AP needs multiple transceivers and each of them is fixed on single channel, while the proposed $\mathrm{CH}-\mathrm{MAC}$ only use one transceiver on AP to realize multi-channel communication.

If each client can select its own available channel without considering if that channel can be used by others, the problem of lacking a common channel will no longer exist. The spectrum variation in one place will not influence the nodes in other locations. Obviously, it is better to leave the job of selecting channel to the channel consumer-client. As well as dealing with spatial and temporal variation of spectrum holes, the heterogenous channel access can reduce conflict significantly by distributing nodes to multiple different channels, and make it possible to conduct communication and channel contention in parallel, which is impossible in traditional homogeneous access. Heterogenous channel access can also provide the opportunity to improve throughput through selecting the most appropriate central frequencies and bandwidths which could be different across multiple clients.

The rest of this paper is organized as follows. Section II details the proposed CH-MAC protocol, and several novel mechanisms are introduced to deal with channel agreement and reduce collision. Section III evaluates the performance improvement through theoretical analysis and simulation. Finally, the paper is concluded in Section IV.

\section{The PROPOSED CH-MAC}

\section{A. Assumptions}

In this paper we design $\mathrm{CH}-\mathrm{MAC}$ without common control channel support. To keep the complexity comparable to existing wireless standards while fully exploiting the flexibility of multiple channels, $\mathrm{CH}-\mathrm{MAC}$ assumes that nodes are equipped with a single half-duplex radio. We focus on spectrum access in this paper, so we assume that AP and clients are capable of identifying available spectrum resources through spectrum sensing or downloading from spectrum database, which is beyond the scope of this paper.

\section{B. Protocol Overview}

AP has to switch among multiple channels in order to communicate with clients distributed among different channels. The channel switch overhead of current software defined radio such as SORA platform is only 0.5-0.7 $\mu s$ [9], which makes the intra-BSS multi-channel access doable. However, there are still a large number of challenges with multi-channel operation. For example, how does a client find AP which could operate on any one of multiple channels? How does AP get the knowledge about which client want to transmit and when to start? These problems will be addressed in the following.

In CH-MAC, each client communicates with AP on the channel that it selects independently based on its local environment and traffic demands. The $\mathrm{CH}-\mathrm{MAC}$ work procedures are divided into two stages. The first stage is called the beacon period (BP), during which channel agreement between AP and client is performed. Data transmission can be conducted in data period (DP) which follows BP. After the channel agreement, AP can get the channel information of each associated client, paving the way for communication in DP. The recurrence of BP as presented in Fig. 2 enables clients to adapt to the temporal variation of spectrum, and the BP period can be adjusted to make a trade off between overhead and adaption to variation. 


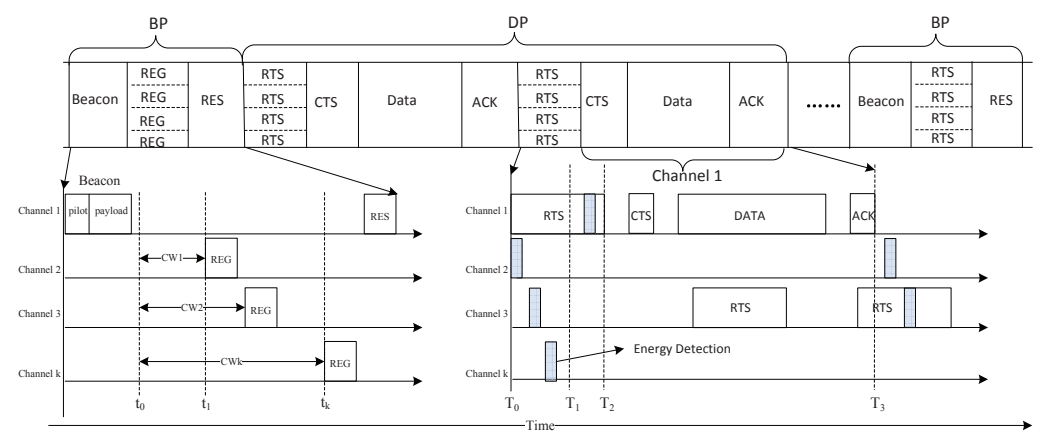

Fig. 2. CH-MAC protocol overview. Channel registration is accomplished in BP for channel agreement, after that, RTS/CTS/DATA/ACK exchange can be conducted on the agreed channel during DP. The shadow block means energy detection of AP for RTS on different channels.

\section{Channel Agreement without Common Control Channel}

AP periodically broadcasts beacons on one of the channels (e.g., channel 1 in Fig. 2) in its own CS, $C S_{A P}$, during BP. $C S_{A P}$ could be changed in different BPs in order to avoid the interference to PUs. Clients scan the spectrum holes to find the beacon, the process of which is similar to the reception of traditional WiFi beacon. Upon receiving the beacon, each client get $C S_{A P}$ and sends the registration (REG) to AP. REG contains the channel information selected by client based on its environment and requirement, which is used for subsequent data transmission. The AP collects all the REGs from multiple clients and get the registration information table which incorporates all associated clients and their channels. After that, data transmissions can be conducted during subsequent DP on different channels.

However, there are some problems to complete the registration process described above. First of all, clients have no idea which channel the beacon is sent on. Thus, the beacon reception needs coarse time synchronization so that clients can scan channels to get beacon at the start of each BP. Intra-cell coarse time synchronization can be achieved after receiving the first beacon just as traditional WiFi, and it's also assumed in existing works [10]. A pilot signal can be attached to the head of beacon such that client can localize the broadcasting channel by performing autocorrelation to pilot. If the duration of pilot $T_{\text {pilot }}$ is not shorter than $\left(T_{\text {scan }}+T_{\text {switch }}\right) \times k$, then clients can localize the beacon message. $T_{\text {scan }}$ is the time for scanning a channel, $T_{\text {switch }}$ is the time for channel switch, and $k$ is the number of channels included in $C S_{A P}$.

The reception of REG is another problem. To be clear, the channel used for sending beacon may be unusable to some of clients in CRNs, therefore client $i$ needs to find a channel in $C S_{A P} \cap C S_{i}$ to send REG. AP then has to detect all the channels included in $C S_{A P}$ to receive REGs which could be transmitted on any channel. One naive detecting sequence could be arranged according to spectrum frequency from low to high. In order to avoid collisions induced by multiple clients transmitting REGs on the same channel, each client should pick a random slot from contention window (CW). AP scans the channels in $C S_{A P}$ one by one, thus there is some blank time on each channel before AP switching into it. For example, the blank time on channel $k$ is $t_{k}-t_{0}$ if AP begins to scan channel 1 at $t_{0}$ and switches into channel $k$ at $t_{k}$. The blank time of each channel can be regarded as $\mathrm{CW}$ which has different numbers of slots on different channels. We use $C W_{i}$ to present the $\mathrm{CW}$ of channel $i$. Each client on channel $i$ will select a slot randomly in $C W_{i}$. Similarly, pilot signal is attached in the head of REG. Clients know that AP will come into channel $i$ at $t_{i}$ due to the time synchronization after receiving beacon, so the pilot will end at $t_{i}$, and REG can be transmitted at this time. Other clients on channel $i$ will refrain if they choose a larger slot. The average of $\mathrm{CW}$ of these $k$ channels is $\left(C W_{1}+C W_{k}\right) / 2$, which is large enough to avoid collision $^{1}$.

At last, a response (RES) from AP to clients is returned. When a client comes into a cell for the first time, or its channel state changes, it performs the registration described above. Other clients that do not want to change their channels have no need to register again. The recurrence of BP make client can choose different channels for communication during different DP, and thus has the ability to adapt to the spectrum variety.

Since the REG only includes client ID and channel information, it would be very small, and the time for transmitting it, $T_{R E G}$, is not too long. The duration of $\mathrm{BP} T_{B P}$ can be deduced by

$$
\begin{aligned}
T_{B P}= & T_{\text {pilot }}+T_{\text {beacon_payload }} \\
& +S I F S+C W_{k}+T_{R E G}+S I F S+T_{R E S}
\end{aligned}
$$

Where $T_{R E S}$ is the time taken for send RES by AP. The period of BP, which should be set properly, not only has to adapt to the change of spectrum, but also can't produce too much overhead. $T_{B P}$ increases in proportion to $\left|C S_{A P}\right|$. If there are 20 channels in $C S_{A P}$, each of which is $6 \mathrm{MHz}$, and $C W_{1}$ is 10 , then $T_{B P}$ will be less than $1.5 \mathrm{~ms}$. The BP period can be set to several seconds, which is enough to adapt the change of spectrum in most white spaces. Thus the BP overhead could be ignored.

\footnotetext{
${ }^{1}$ In TV white space, it could be almost 50 if there are $206 \mathrm{MHz}$ channels in $C S_{A P}$ and a slot is $9 \mu \mathrm{s}$.
} 


\section{Data Transmission}

When the beacon period has finished, every client switches into its own registered channel, and the communication between AP and clients could be carried out on these channels during DP. The communication is built on two way RTS/CTS handshake which acts like the DCF of 802.11 MAC protocol, except that it's accomplished on multiple channels. In the downlink, AP can switch into the target channel actively to send data to clients since the channel information of each client has been collected during BP.

However, that is not easy in uplink: AP needs to know which clients are going to send data to it, as well as the start time of transmission. Thus, in following part we primarily focus on uplink communication. In order to address that challenge, AP should be capable of receiving multiple RTSs sent on different channels simultaneously under the condition of no strict time/frequency synchronization with clients. This is impossible with traditional RTS receiving mechanism. Therefore, we adopt subcarrier coding (SC) to encode RTS so that the reception of RTS no longer requires time and frequency synchronization. This is very important in multichannel scenario for the reason that AP may be on a different channel when a client is transmitting RTS. The feasibility and reliability of subcarrier coding has been demonstrated in [11] with SORA platform. Different from the conventional RTS receiving scheme, SC uses the on/off status of subcarriers to encode message and uses energy detection to decode it. This is feasible because each subcarrier of a channel can be turned on or off individually in OFDM system which is widely adopted in current communication system. For example, 11010 can be used to represent a RTS, which denotes 5 subcarriers with the first, second and fourth subcarriers turned on and others turned off. There are two advantages brought by energy detection: one is that the time taken for decoding RTS is very short, the other is that time and frequency synchronization is no longer required. Only $1.6 \mu \mathrm{s}$ is required to scan a $20 \mathrm{MHz}$ channel with the SORA platform [9]. Therefore, if a client sends RTS lasting for $40 \mu s, 400 \mathrm{MHz}$ spectrum band can be scanned during that time. In other words, with the subcarrier coding, AP can simultaneously receive up to 20 RTSs sent in $400 \mathrm{MHz}$ spectrum bands respectively if each RTS occupies $20 \mathrm{MHz}$ bandwidth. In order to increase the success rate of energy detection, each channel could be scanned several times. However, the scanning time, $T_{\text {scan }}$, of a channel is still very short.

The lower right of Fig. 2 presents the process of receiving RTS. AP can scan $n$ channels registered from channel 1 to channel $n$ during DP when it's idle. In the worst case, a client on channel 1 transmits RTS at $T_{0}$, but at that moment, AP just left channel 1 and switches into channel 2. However, RTS of client 1 can still be received as long as $T_{R T S} \geq\left(T_{\text {scan }}+\right.$ $\left.T_{\text {switch }}\right) \times n$, where $T_{R T S}$ is the duration of RTS signal, which is $T_{2}-T_{0}$ in Figure 2. We note that before AP switching into channel 1 at $T_{1}$, the RTS signal has been transmitted for a period of time, but RTS can still be received correctly due to that energy detection is used to decode information.

\section{E. Discussions}

The on/off status of subcarriers are used to encode RTS which needs only to contain the client ID. AP need only to know which client wants to send message. The ID could be client's MAC address, but it's still large considering that 48 bits should be encoded and dozens of subcarriers are required. The number of subcarriers of a channel is limited ${ }^{1}$. AP can assign a unique number to each client as its ID, which is mapped to MAC address. Ten bits or less would be enough considering that the number of clients associated to an AP is usually less than one thousand. This means that only parts of subcarriers of a channel are used by one client to encode RTS, and the rest could be exploited by others without collision. Without the need for learning which subcarriers are used in advance, AP just switches into a channel registered during BP and detects the energy of each subcarrier.

To encode RTS, channel is partitioned into multiple subcarrier groups (SG), each of which is used to transmit one RTS by client. Channel with more subcarriers can afford more SGs and accommodate more RTSs without collision. If a SG is selected by two or more clients, there would be conflict and RTS can't be received. But this type of collision can be detected by appropriate coding scheme[11].

A client with data to send picks a slot randomly from $\mathrm{CW}$ and wait for that slot before attempting to access the medium. When several clients who choose the same SG are attempting to transmit, the client that picks the first slot wins. If AP is receiving frame on channel 1 during $T_{1}-T_{3}$ as presented in Fig. 2, the RTS sent by another client on channel 3 can't be received. Therefore, the client on channel 3 should send RTS multiple times until it is received successfully. The lost of RTS is not only caused by collision, hence it should not trigger back off, and the contention window won't be increased when clients try to resend RTS. A Channel will not be occupied by one client consistently even it may send RTS several times because NAV (Net Allocation Vector) will be only included in CTS, not RTS since the RTS can not carrier too much information. Getting rid of NAV from RTS has no effect to hidden terminals which can't hear RTS anyhow. Clients attempting to access AP will stop sending RTS for a while when it doesn't receive CTS after transmitting one or two RTSs to avoid saturating channel.

All clients have been shunted twice by the algorithm proposed which reduces conflict significantly. At the first time, clients are shunted to different channels by channel selection during BP. At the second time, the clients on a same channel are shunted to different SGs. However, conflict might still occur. So we adopt some effective mechanisms to further eliminate collision. Only brief presentation is made due to space constraints.

At first, coordination among clients can be conducted in parallel with communication. For example, when AP is com-

\footnotetext{
${ }^{1}$ There are 64 subcarriers for $20 \mathrm{MHz}$ channel in $802.11 \mathrm{~g}$, and 1440 subcarriers for 802.22 .
} 
municating with one client on channel 1 during $T_{1}-T_{3}$ in Fig. 2, other clients can transmit RTS on channel 4. After several attempts at coordination during communication, each client can choose an SG with the least number of clients. AP can detect these SGs simultaneously on a channel, so if there is no collision on at least one of these SGs, the RTS encoded with this collision-free SG can be received.

Secondly, even if there is collision on every SG of a channel, the overhead introduced by collision can still be minimized. AP can be aware of the collision and overview the crashed RTSs on all SGs of a channel. Rather than waiting on current channel until someone reselects a slot in a large contention window after DIFS, AP can switch into another channel to keep on communication after $T_{\text {scan }}$ slipped. Through this mean, the overhead caused by collision could be cut down in a great extent.

\section{PERFORMANCE ANALYSIS AND SIMULATION}

\section{A. Performance analysis}

The performance of CH-MAC is evaluated with modified Bianchi model which is accurate in the assumption of ideal channel conditions. We will analytical evaluate the saturation throughput. As described above, a client with data to send will select a SG of a registered channel to transmit RTS. Besides that, it also need to choose the backoff time uniformly in the range $(1, w-1)$. The value of $\mathrm{CW}, w$, is constant no matter how many times a RTS has been transmitted. Since the value of the backoff counter doesn't depend on its transmission history, every client transmits RTS on its SG with the probability $\pi=1 / w$ in a generic (i.e., randomly chosen) slot time. Then, by studying the events that could occur within a generic slot time, we can express the throughput of $\mathrm{CH}-$ MAC.

Unlike the traditional IEEE 802.11 single channel access, all nodes in a BSS are located on different channels. So appropriate modulation to the Bianchi model is required to evaluate CH-MAC. Since an AP can only stay on one channel at a time, we just need to consider the events on that channel in a slot. The backoff time counter of each client decreases at the beginning of each slot time. When the SG selected by a client is sensed busy, the decrement is stopped. Therefore, the time interval between two consecutive slot time beginnings may be much longer than the slot time size, as it may include a packet transmission. Therefore, with the term slot time we will refer to either the regular constant slot size $(9 \mu \mathrm{s}$ in $802.11 \mathrm{~g})$, and the (variable) time interval between two consecutive backoff time counter decrements.

The key approximation in our model is that, at each transmission attempt, and regardless of the number of retransmissions suffered, each packet collides with constant and independent probability $P_{c}^{i}$ on the $i$ th SG of a channel. the probability $P_{c}^{i}$ that a transmitted packet encounters a collision, is the probability that at least two of the clients transmit on the same SG $i$. Let $P_{t r}^{i}$ be the probability of at least one transmission in the considered slot time, it can be deduced as $P_{t r}^{i}=1-(1-\pi)^{N_{i}}$
Here $N_{i}$ is the number of clients choosing the $i$ th SG on the considered channel. The probability $P_{s}^{i}$ that a transmission on $i$ th SG is successful is given by the probability that exactly one client transmits on $i$ th $\mathrm{SG}$, conditioned on the fact that at least one client transmits, i.e.,

$$
P_{s}^{i}=\frac{N_{i} \pi(1-\pi)^{N_{i}-1}}{P_{t r}^{i}}
$$

Each client chooses a SG with least number of clients based on its observation to reduce collision. So we can assume $N_{i}$ is equally with $N / k$, while there are $N$ clients on the considered channel which is divided into $k$ SGs. Thus $P_{c}^{i}$ can be yielded

$$
\begin{aligned}
P_{c}^{i} & =P_{t r}^{i}\left(1-P_{s}^{i}\right) \\
& =1-(1-\pi)^{N / k}-N / k \pi(1-\pi)^{N / k-1}
\end{aligned}
$$

Conflict taking place on the $i$ th SG of a channel doesn't mean that AP can't receive RTS on that channel. Realize that AP can detect all of the $k$ SGs of a channel to receive multiple RTSs simultaneously, so if there is no collision on at least one of $k$ SGs , RTS transmitted on current channel can still be received accurately. Hence the probability of collision on a channel $P_{c}$ is obtained by $P_{c}=P($ conflict in every $S G)=P_{c}^{i^{k}}$. The probability that a transmission occurring on a channel is successful is given by

$$
P_{s}=1-P_{i d}-P_{c}=1-(1-\pi)^{N}-\left(P_{c}^{i}\right)^{k}
$$

Where $P_{i d}$ is the probability that there is no client transmitting in the slot on considered channel.

The saturation throughput, which is the average information payload transmitted in a slot time over the average duration of a slot time, can be computed as follows:

$$
\begin{aligned}
\Gamma & =\frac{E[\text { Payload information transmitted in a slot time }]}{E[\text { Duration of slot time }]} \\
& =\frac{P_{s} L+P_{c} L_{c}}{P_{s} T_{s}+P_{c} T_{c}+P_{i d} T_{i d}}
\end{aligned}
$$

Where $L$ is the average packet payload size; $T_{s}$ is the average time needed to transmit a packet of size $L ; T_{i d}$ is the duration of the idle period (a single slot time); $T_{c}$ is the average time spent in the collision. When conflict happen on current channel, each client on this channel will freeze its backoff counter for $T_{c}$. In order to have a minimum amount of collision overhead, AP will leave current channel to continue scanning next channel after $T_{\text {scan }}$ has elapsed. Thus the message of size $L_{c}$ can be transmitted on another channel during $T_{c}$. We can deduce $L_{c}$ by $L_{c}=\left(T_{c}-T_{r}\right) \times \Gamma$, Where $T_{r}$ is the time taken for finding a RTS on other channel by AP.

In the period of $\left(T_{c}-T_{r}\right)$, message can still be transmitted.

$$
\begin{aligned}
T_{r}= & \sum_{k=1}^{d-2} k T_{\text {scan }} P_{s} r+T_{c}\left(1-P_{s} r\right)^{d-1} \\
= & T_{\text {scan }}\left(\frac{1-\left(1-P_{s} r\right)^{d-1}}{P_{s} r}-(d-1)\left(1-P_{s} r\right)^{d-1}\right) \\
& +T_{c}\left(1-P_{s} r\right)^{d-1}
\end{aligned}
$$


TABLE I

SYSTEM PARAMETERS USED TO OBTAIN NUMERICAL RESULT

\begin{tabular}{|c|c|}
\hline packet payload & 8184 bits \\
\hline MAC header & 272 bits \\
\hline PHY header & 128 bits \\
\hline ACK & 112 bits + PHY header \\
\hline CTS & 112 bits + PHY header \\
\hline Propagation & $1 \mu \mathrm{s}$ \\
\hline Slot Time & $9 \mu \mathrm{s}$ \\
\hline SIFS & $10 \mu \mathrm{s}$ \\
\hline DIFS & $28 \mu \mathrm{s}$ \\
\hline Bit rate & $54 \mathrm{Mbps}$ \\
\hline
\end{tabular}

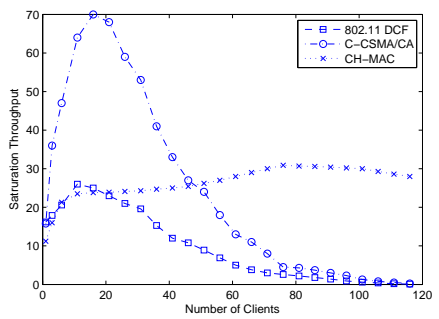

Fig. 3. The saturation throughput of different protocols

Where $r=\frac{R T S}{R T S+D I F S}$ is the average probability of receiving RTS on any channel, conditioned on the fact that at least one client transmits. $d$ is the maximum number of channels detected by AP during $T_{c}$ which can be calculated by $d=\frac{T_{c}}{T_{s c a n}+T_{\text {switch }}}, T_{s}$ and $T_{c}$ can be calculated with

$$
\left\{\begin{aligned}
T_{s}= & R T S+S I F S+\sigma+C T S+S I F S+\sigma+H+L \\
& +S I F S+A C K+D I F S+\sigma \\
T_{c}= & R T S+D I F S+\sigma
\end{aligned}\right.
$$

where $H, L$, and $A C K$ are the transmission times needed to send the packet header, the payload, and the acknowledgment, respectively. $\sigma$ is the propagation delay.

At last, the saturation throughput of network $\Gamma$ can be deduced as follows:

$$
\Gamma=\frac{P_{s} L}{P_{s} T_{s}+P_{c} T_{r}+P_{i d} T_{i d}}
$$

\section{B. Simulation}

We simulate the saturation throughput of CH-MAC in different scenarios. The values of the parameters used to obtain numerical results are summarized in Table I. Owing to space constrain, only part of the simulation results are presented.

In the following we compare the performance of $\mathrm{CH}-\mathrm{MAC}$ with traditional 802.11 DCF and C-CSMA/CA in terms of throughput. The throughput of DCF drops by $83 \%$ when there are 50 clients, and it's almost zero when 100 clients associate to AP. The large number of clients crammed together on a channel lead to serious collision, and RTS could hardly be received correctly. The AP has three transceivers in CCSMA/CA protocol, thus the throughput is higher than others when the number of clients is small. But the throughput decrease largely with large number of clients due to each

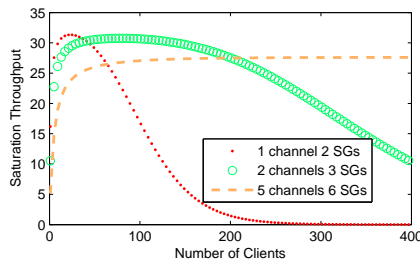

Fig. 4. The saturation throughput obtained at the condition of different number of channels and SGs

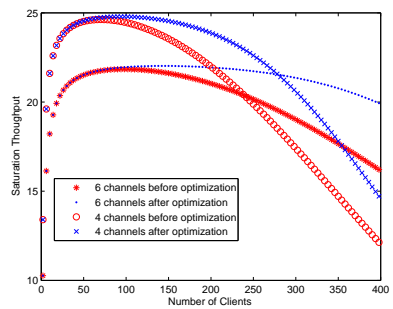

Fig. 5. Benefit brought by the optimization mechanism, the two blue lines and two red lines are throughput without and with the optimization respectively

transceiver if fixed on a single channel. With more channels and SGs, clients can select different channels and interference can be reduced.

Figure 4 shows the throughput strongly depend on the number of channels that AP needs to detect during DP. More stable throughput can be obtained with more channels and SGs as the number of clients increases. Doubling the number of SGs can reduce conflict and increase throughput considering that a SG with fewer clients can be selected to transmit RTS. More channels and SGs can provide more stable throughput even clients increase largely because these clients can be distributed on different channels and SGs. But there is a shortcoming when more channels are used. When there are small number of clients, less than 5 clients, the throughput of network is not ideal. This is because that AP needs to detect multiple channels even there is no client on some of them. That will create a certain number of overhead.

Heterogenous multi-channel access mechanism can decrease collision and improve throughput significantly even large number of clients exist as shown in Figure 4. But conflict is inevitable when the number of clients surpasses the product of channel number and SG number. A new method as described in last section has been taken to further reduce the impact of conflict: AP will switch into another channel rather than stay on a channel that exists collision. Figure 5 illustrates that this mechanism can enhance $20 \%$ of the throughput when the conflict is serious. This test has been conducted with 4 and 6 channels respectively, and the more channels are used, the more obvious the advantage is. This is because AP can find RTS with bigger probability with more channels during $T_{c}$.

We evaluate the influence of $\mathrm{CW}$ value on throughput, which is shown in Figure 6. The CW value is set 10, 15, 20, and 40 respectively. The performance is better with small $\mathrm{CW}$ under low number of clients. But larger $\mathrm{CW}$ performs better 


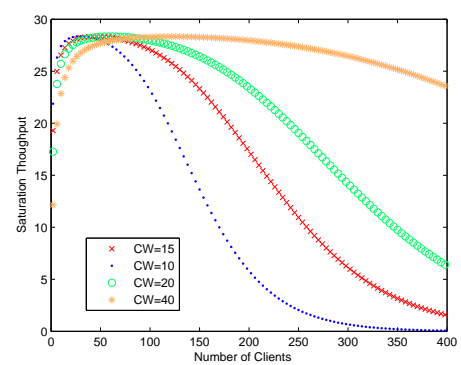

Fig. 6. The saturation throughput obtained at the condition of different contention windows

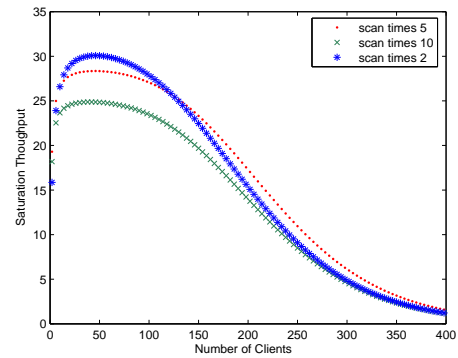

Fig. 7. The saturation throughput obtained at the condition of different channel scanning times

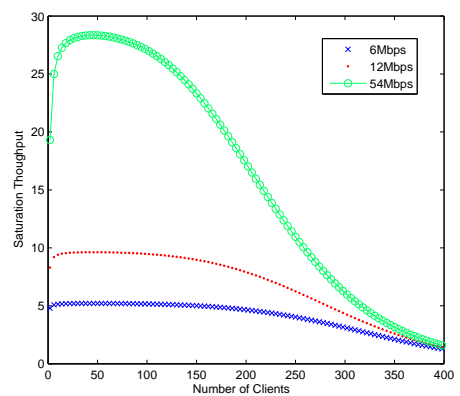

Fig. 8. The saturation throughput obtained at different rates

under high number of clients. This is easy to understand that larger $\mathrm{CW}$ can accommodate more clients than small $\mathrm{CW}$, but the overhead could be large too. An adaptive CW setup can improve the performance of $\mathrm{CH}-\mathrm{MAC}$, and it will be as future work.

RTS is decoded with energy detection, therefore more detection times can increase the detection ratio, but this will increase the scanning time on a channel. When the scanning times on each channel is 2,5 , and 10 , the RTS detection ratio is about $0.6,0.8$ and 0.96 respectively when $S N R=20 \mathrm{~dB}$. Fig.7 shows the influence of scanning times to throughput. When there are large number of clients, more scanning times works better than less scanning times because the RTS without collision (that is rare with more clients) should be catched as early as possible.

We also test the performance of $\mathrm{CH}-\mathrm{MAC}$ at different physical rates. The curves in Fig. 8 correspond to different throughput values at different data rates. The higher rate is more sensitive to collision for the reason that the protocol control overhead has more influence on throughput.

\section{CONCLUSION}

This paper analyzes the problems of traditional homogeneous channel access in cognitive WLANs. By designing a novel MAC protocol, each client can access its best channel without the influence of other nodes in the same cell. Collision is also reduce significantly through transforming conventional time domain contention into frequency-time contention. We believe that $\mathrm{CH}-\mathrm{MAC}$ is innovative in many respects and opens up new way of spectrum exploitation in cognitive WLANs. We only present the simulations of this protocol, and the prototype system are being developed which has not been included in this paper.

\section{ACKNOWLEDGMENT}

TThis research is partially supported by the National Science Foundation of China under Grant No. 61070211, No. 61003304 and No 61070201, grants from the Ph.D. Programs Foundation of Ministry of Education of China No. 20114307120003, 2010430711004

\section{REFERENCES}

[1] P. Bahl, R. Chandra, T. Moscibroda, R. Murty, and M. Welsh, "White space networking with wi-fi like connectivity," in ACM SIGCOMM, vol. 39, 2009, pp. 27-38.

[2] C. Ghosh, S. Roy, M. Rao, and D. Agrawal, "Spectrum occupancy validation and modeling using real-time measurements," in Proceedings of the 2010 ACM workshop on Cognitive radio networks, 2010, pp. 25-30.

[3] D. Chen, S. Yin, Q. Zhang, M. Liu, and S. Li, "Mining spectrum usage data: a large-scale spectrum measurement study," in ACM Mobicom, 2009 , pp. $13-24$.

[4] IEEE 802.22 Working Group. [Online]. Available: http://www.ieee802.org/22/.

[5] J. Jia, Q. Zhang, and X. Shen, "Hc-mac: A hardware-constrained cognitive mac for efficient spectrum management," Selected Areas in Communications, IEEE Journal on, vol. 26, no. 1, pp. 106-117, 2008.

[6] C. Cordeiro and K. Challapali, "C-mac: a cognitive mac protocol for multi-channel wireless networks," in IEEE DySPAN, 2007, pp. 147-157.

[7] J. So and N. Vaidya., "Multi-channel mac for ad hoc networks: Handling multi-channel hidden terminals using a single transceiver," in $A C M$ MobiHoc, 2004, pp. 222-233.

[8] K. Tan, J. Fang, Y. Zhang, S. Chen, L. Shi, J. Zhang, and Y. Zhang, "Fine-grained channel access in wireless lan," in ACM SIGCOMM, vol. 40, no. 4, 2010, pp. 147-158.

[9] K. Tan, H. Liu, J. Zhang, Y. Zhang, J. Fang, and G. Voelker, "Sora: high-performance software radio using general-purpose multi-core processors," Communications of the ACM, vol. 54, no. 1, pp. 99-107, 2011.

[10] Y. Yuan, P. Bahl, R. Chandra, P. Chou, J. Ferrell, T. Moscibroda, S. Narlanka, and Y. Wu, "Knows: Cognitive radio networks over white spaces," in IEEE DySPAN, 2007, pp. 416-427.

[11] D. Chen, J. Zhang, and Q. Zhang, "Subcarrier coding: A fast multichannel coordination approach in cognitive radio networks," in $A C M$ Mobicom, Poster, 2011, pp. 37-48.

[12] Q. Zhang, F. H. Fitzek, and Iversen, "Cognitive radio mac protocol for wlan," in IEEE PIMRC, 2008, pp. 1-6. 